\title{
STRUCTURAL CHANGES OBSERVED IN MICE LIVER DUE TO CHRONIC EXPOSURE OF 1900-2200 MHZ RADIATIONS EMITTED FROM MOBILE PHONE
}

\author{
N Mugunthan *1, J Anbalagan 2, S Meenachi ${ }^{3}$, S Rajanarayanan ${ }^{4}$. \\ ${ }^{* 1}$ Professor, Department of Anatomy, Sree Mookambika Institute of Medical Sciences, Kulasekharam, \\ India. \\ 2 Professor, Department of Anatomy, Mahatma Gandhi Medical College \& Research Institute, \\ Puducherry, India. \\ ${ }^{3}$ Deputy Director of Health Services, Paramakudi HUD, Tamil Nadu. \\ ${ }^{4}$ Professor \& H.O.D, Department of Bio-technology, St.Michael College of Engineering \& Technol- \\ ogy, Kalayarkoil, India.
}

\section{ABSTRACT}

\begin{abstract}
Background: Rapid developments in the telephone technology made the communication faster and easier. The duration of the use of mobile phones also increased beyond the limitation, and has raised public concerns about their safety. This study is designed to investigate the long term exposure of $1900 \mathrm{MHz}$ to $2200 \mathrm{MHz}$ radiations effect emitted from the third generation (3G) mobile phone in mice liver at histological and biochemical level.

Materials and Methods: With approval from institutional animal ethics committee, 36 mice were exposed to $3 G$ mobile phone radiation, 48 minutes per day for a period of $30-180$ days. The control group (36 mice) was kept under similar conditions without $3 G$ exposure. The animals were sacrificed and the liver was removed from first month to six months period. $1 \mathrm{ml}$ of blood was collected for SGOT and SGPT analysis. Liver was processed for antioxidant enzyme activity - SOD \& GPx, and for routine histological study. The serial sections were analysed under microscope for structural changes. Two groups' data were compared and analysed statistically.

Results: In comparision to control group the liver of $3 \mathrm{G}$ exposed groups showed the following changes: parenchyma and the periportal regions were infiltrated with inflammatory cells. Focal haemorrhages in the liver parenchyma dilated and congested hepatic sinusoids. Swollen hepatocytes with pyknotic nuclei and vacuolations in the cytoplasm were the significant changes. Biochemical parameters did not show any variable difference.

Conclusion: The long term exposure of ultra-high frequency radiation emitted from a $3 G$ mobile phone could cause microscopic structural changes in the liver.

KEY WORDS: $3 G$ mobile phone, liver, radiation.
\end{abstract}

Address for Correspondence: Dr.N.Mugunthan. MBBS., M.S., D.N.B., PhD., Professor, Department of Anatomy, Sree Mookambika Institute of Medical Sciences, Padanilam, Kulasekharam, Kanyakumari District, Tamil Nadu, India, Pincode -629161. Mobile: +919443118932

E-Mail: dr.mugunth111@gmail.com

Access this Article online

Quick Response code

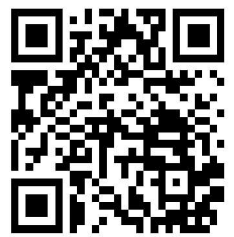

DOI: $10.16965 /$ ijar.2018.438

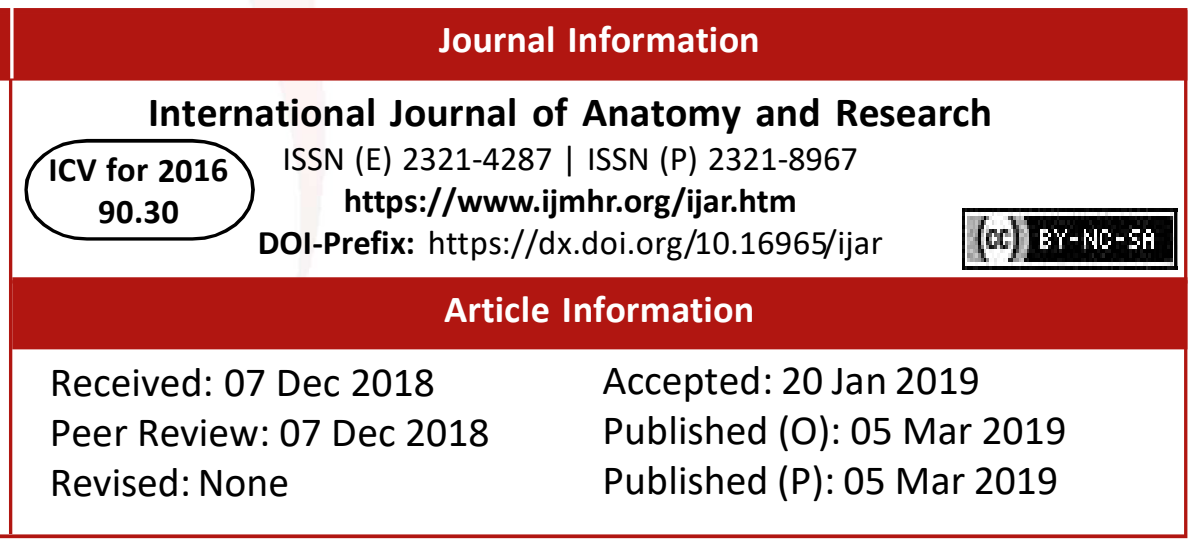

INTRODUCTION

Rapid developments in telephone technology made the communication faster and easier. The proliferation and the introduction of new 
generation mobile phones led to a widespread concern of public safety. The usage of mobile phone by individuals of all ages, young and the old, the increased usage of mobile phones beyond the excessive limits, have raised public concerns about their safety on public health.

The third generation mobile phones (3G) operate at a frequency between 1900-2200 $\mathrm{MHz}$, and have been designed to carry voice communications, video games, playback, email access, internet browsing, video telephony, high speed data access and music downloads [1]. While using a mobile phone, the user's body is exposed to electromagnetic fields (EMFs) emitted both from mobile phone and the base stations. Mobile phone in operation emits radiofrequency electromagnetic field (RF-EMF) usually in the form of a continuous wave (CW), sometimes pulse-modulated and occasionally amplitude-modulated at extremely low frequencies. Radiation energy is absorbed into human body by three mechanisms: (i) Aerial effect- body receives and absorbs the RF signal depending upon the size of the body part and wavelength of the signal (ii) Coupling the RF signal with the tissue and (iii) Resonant absorption [2,3]. Most of the energy emitted from mobile phones is absorbed into the user's body, particularly in the head region, thus producing heat stress, non-thermal stress in the form of releasing free radicals and alteration in the enzyme reaction, thereby compromising the immune system.

Earlier reports have shown that the exposure to mobile phone radiation induced tissue damage at the molecular level. The damages resulted in single and double strand DNA breakages [4], reduction of Purkinje cell number in the adult female rat cerebellum [5], significant reduction in sperm count [6], reduction in serum testosterone levels [7], short term memory effects in mice [8] and people residing near the base station were psychologically stressed more [9]. Similarly researchers reported that short term exposure of mobile phone radiation induced damages in the kidney: atrophied glomeruli, leukocytic infiltrations and extravasation of blood cells between the kidney tubules $[10,11]$, congested and dilated renal veins. And also, epithelial cells of the renal tubules showing cytoplasmic vacuolations with pyknotic nuclei [12], obstruction of some convoluted tubules, fibrotic accumulations around the convoluted tubules [13] and $d$ isruption of the brush border in the proximal convoluted tubules $[14,15]$ were also cited.

Research studies on EMF exposed liver revealed intense inflammatory responses around the central vein, swollen hepatocytes and highly vacuolated cytoplasm [10], congestion of the hepatic sinusoids and the central vein [11], pyknotic nucleus with necrosis of endothelial cells and apoptosis. Hepatocytes and Kupffer cells were seen in apoptosis form [16].

In contrary to the above findings, some reports stated that, there were no adverse biological effects of exposure to the non-ionizing radiation from the mobile phone: no breakage of double stranded DNA or effects on chromatin of rat brain [17], no effect on mouse embryonic lens development [18], no effect on psychomotor performance [19] and no histological changes on rat testis [20].

Keeping in view that, the increased number of mobile phone calls per day, length of each call, amount of time of usage of mobile phones and casual erection of the base stations in the residential areas, this study is designed to investigate the dose dependant effects of chronic exposure of $3 G$ mobile phone induced EMFs in mice liver with reference to the structural changes at histological and biochemical level.

The contradictory scientific reports upon the effects of mobile phone radiation on biological tissues raised much concern on human health. Liver is the largest solid organ and handles various metabolisms and body's detoxification system. Liver being a solid organ, it absorbs more radiation. The rapid proliferation of wireless communication has prompted us to undertake the present study to evaluate the possible effects of liver damage following chronic exposure to ultra high frequency radiation emitted from $3 \mathrm{G}$ mobile phone on mice model with reference to structural changes at histological and functional changes at biochemical level.

\section{MATERIALS AND METHODS}

This study was approved by the Institutional Animal Ethics Committee of Mahatma Gandhi 
Medical College and Research Institute, Puducherry. Seventy-two neonatal albino mice procured from the King Institute of Preventive Medicine and Research, animal section, Chennai, constituted the material for experimental study.

New born mice were randomly divided into two independent groups; control and 3G exposed. Mice were kept in cages in the central animal house at $22 \pm 1^{\circ} \mathrm{C}, 60 \%$ relative humidity, adequate ventilation; twelve hours of illumination alternated with twelve hours of darkness. During the study, all the animals were fed with laboratory diet and water ad libitum. Thirty six mice were exposed to $1900-2200 \mathrm{MHz}$ frequency radiation emitted from $3 \mathrm{G}$ mobile phone and thirty six mice were control.

The roof of the mice cage was designed to hang the $3 \mathrm{G}$ mobile phone from a distance of five $\mathrm{cm}$ from the floor. This distance allowed the mice to move freely and to avoid direct thermal injury. The $3 \mathrm{G}$ mobile phone in non-vibrating, silent, do not disturb (DND) with auto answer mode activated, was kept hanging inside the mice cage. $3 \mathrm{G}$ mobile phone operates on a frequency bandwidth of $1900-2200 \mathrm{MHz}$ and with the power of $2 \mathrm{~W} / \mathrm{Kg}$. The highest specific absorption rate (SAR) value for this standard handset was $1.69 \mathrm{~W} / \mathrm{Kg}(10 \mathrm{gm})$ and this SAR value was within the limit of the International Commission on Non-lonizing Radiation Protection (ICNIRP) recommendation.

The mobile phone kept inside the mouse's cage was rung upon from another $3 \mathrm{G}$ mobile phone for every half an hour, each call lasting for two minutes. Exposure time was forty eight minutes per day for a twelve hour periods from 8.00 AM8.00 PM. The total duration of exposure was thirty to one hundred and eighty days. A Radiofrequency meter (RF) was used to measure the amount of radiation exposed in $3 G$ experimental groups. The control group of thirty six mice was kept under similar conditions without $3 \mathrm{G}$ exposure. Six mice were sacrificed at the end of $30,60,90,120,150$ and 180 days of exposures in the experimental groups after twenty four hours of last exposure. Equal numbers of control mice were sacrificed on similar experimental design.

During the time of sacrificing the mice, $1 \mathrm{ml}$ of Int J Anat Res 2019, 7(1.3):6254-60. I ISSN 2321-4287 blood was collected for SGOT (Serum Glutamic Oxalo acetic Transaminase) and SGPT (Serum Glutamate Pyruvate Transaminase) analysis. SGOT and SGPT were measured using Cobas c 111 analyser. Liver was removed by careful dissection and a portion of the liver was preserved for antioxidant enzyme activity -SOD (Superoxide Dismutase) and GPx (Glutathione Peroxidase). SOD and GPx activity in the liver tissues was measured by quantitative calorimetric determination method using EnzyChrom Superoxide Dismutase and Glutathione Peroxidase Assay Kit (BioAssay Systems). Remaining part of the liver was fixed in $4 \%$ formalin solutions for twenty four hours, and then processed by routine histological procedures. Liver tissues were sectioned at five microns, stained with Haematoxylin \& Eosin and PAS (periodic acid Schiff stain). Liver sections were analysed from random slide, random sections and random field under the light microscope for histomorphometric parameters and structural changes.

Statistical analysis: The Mann Whitney $U$ test was used for comparing control and $3 \mathrm{G}$ exposed groups. $P$ value $\leq 0.05$ was considered statistically significant. Data were analyzed using SPSS 16 software.

\section{RESULTS}

Histological study: The liver sections of the control group showed normal architecture of hepatic lobule, with central vein in the centre of lobule and the portal triads at the periphery. Polygonal shaped hepatocytes were arranged in sheets with acidophilic cytoplasm. Rounded basophilic nuclei occupied the centre of the cell. Few hepatocytes showed two or more nuclei with one or two nucleoli. The hepatic sinusoids were lined by endothelial cells; stellate shaped macrophages-Kupffer cells were also seen. The hepatocyte cytoplasm appeared magenta under PAS stain. The nuclei appeared as basophilic round body. Glycogen areas were seen with intense magenta (Fig.01 -1a, 2a, 3a, 4a, 5a \& $6 a)$.

In 3G mobile phone exposure group, the liver sections showed infiltration with lymphocytes and neutrophils at the peripheral regions indicating mixed type of inflammation in the 
Fig. 1: Photomicrograph showing liver of control and 3G exposed mice: 1a, 2a,3a,4a,5a \& 6a - control liver from 1to 6month, 1b,2b,3b,4b,5b \& 6b -3G exposed liver from 1to 6month, H\&E -haematoxylin \& eosin stain, PAS - periodic acid Schiff stain, Square dotted line - portal triad, PV- portal vein, BD - bile duct, HA - hepatic artery, CV - central vein, Blue dotted circle - haemorrhages in the parenchyma, Black \& white arrow -sinusoids, Blue arrow head cytoplasmic vacuolations, Yellow arrow head - pyknotic nuclei, Yellow dotted circle- inflammatory infiltration, Blue asterix - increased sinusoidal space, 400X magnification.
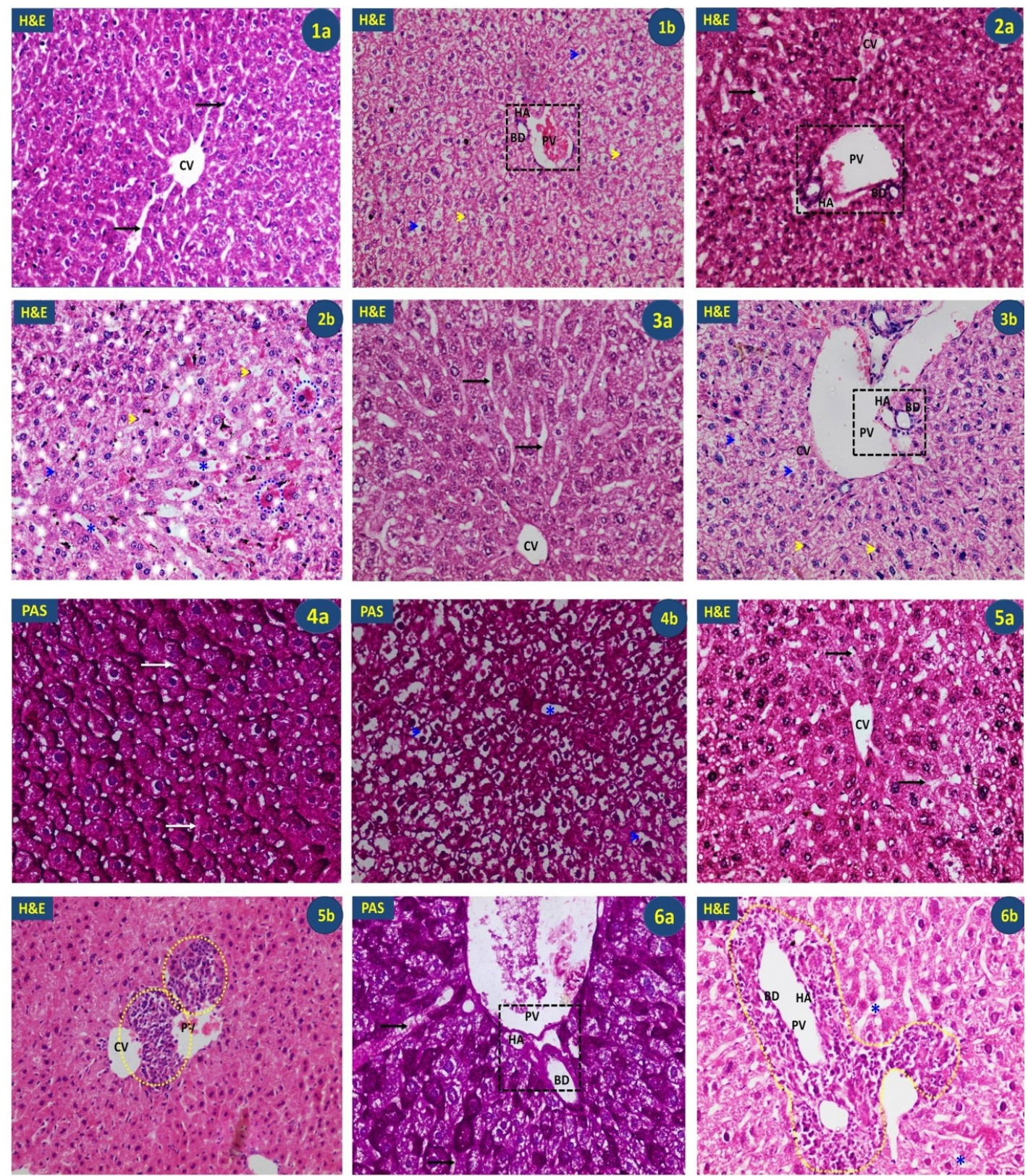

fourth and fifth month period. Focal haemorrhages (hemosiderin granules + ) were found within the liver parenchyma. Hepatic sinusoids appeared congested and dilated. Vacuolations were present in the cytoplasm of hepatocytes, which appeared as irregular foamy spaces. Many hepatocytes appeared swollen with pyknotic nuclei. Under PAS stain, the cytoplasmic vacuolations appeared as empty spaces and pyknotic nuclei as dark blue stained clumped irregular dense structures (Fig.01-1b, 2b, 3b, $4 b, 5 b \& 6 b)$.

\section{Biochemical study}

SGOT \& SGPT: Comparison between control and $3 \mathrm{G}$ exposed groups by Non-parametric comparison using Mann - Whitney $U$ test showed no significant difference in the mean SGOT \& SGPT ( $p$ value $>0.05$ ) [Table.01]

Antioxidant enzyme activity - SOD \& GPx: There was no significant difference in the mean SOD \& GPx activity ( $p$ value $>0.05$ ) in the comparison between control and $3 G$ exposed groups by Non-parametric comparison using Mann - Whitney U test. [Table. 1] 
Table 1: shows the mean \pm SD of SGOT, SGPT, SOD and GPx of control and $3 G$ exposed mice.

\begin{tabular}{|c|c|c|c|c|c|}
\hline $\begin{array}{c}\text { Group n=18 } \\
\text { in each group }\end{array}$ & Month & $\begin{array}{c}\text { Mean } \pm \text { SD } \\
\text { SGOT (mg/dl) }\end{array}$ & $\begin{array}{c}\text { Mean } \pm \text { SD } \\
\text { SGPT (mg/dl) }\end{array}$ & $\begin{array}{c}\text { Mean } \pm \text { SD } \\
\text { SOD activity } \\
\text { (unit/gram protein) }\end{array}$ & $\begin{array}{c}\text { Mean } \pm \text { SD } \\
\text { GPx activity } \\
\text { (unit/mg protein) }\end{array}$ \\
\hline \multirow{5}{*}{ Control } & $\mathbf{1}$ & $177.00 \pm 18.35$ & $65.67 \pm 20.55$ & $0.30 \pm 0.00$ & $18.22 \pm 0.00$ \\
\cline { 2 - 6 } & $\mathbf{2}$ & $170.00 \pm 22.27$ & $49.33 \pm 17.21$ & $0.33 \pm 0.00$ & $19.41 \pm 0.00$ \\
\cline { 2 - 6 } & $\mathbf{3}$ & $162.00 \pm 32.18$ & $52.67 \pm 17.00$ & $0.31 \pm 0.00$ & $20.01 \pm 0.00$ \\
\hline & $\mathbf{5}$ & $159.00 \pm 34.39$ & $68.67 \pm 15.01$ & $0.39 \pm 0.00$ & $18.53 \pm 0.00$ \\
\hline \multirow{5}{*}{ 3G exposed } & $\mathbf{6}$ & $173.33 \pm 18.03$ & $71.00 \pm 15.71$ & $0.40 \pm 0.00$ & $18.39 \pm 0.00$ \\
\hline & $\mathbf{2}$ & $222.33 \pm 16.25$ & $79.00 \pm 26.28$ & $0.72 \pm 0.00$ & $67.71 \pm 0.00$ \\
\hline & $\mathbf{3}$ & $223.00 \pm 15.13$ & $60.00 \pm 28.00$ & $0.80 \pm 0.00$ & $70.02 \pm 0.00$ \\
\hline & $\mathbf{4}$ & $213.67 \pm 11.93$ & $76.67 \pm 18.03$ & $0.70 \pm 0.00$ & $64.00 .52 \pm 0.00$ \\
\hline & $\mathbf{5}$ & $220.33 \pm 11.23$ & $84.33 \pm 39.80$ & $0.82 \pm 0.00$ & $68.04 \pm 0.00$ \\
\hline
\end{tabular}

\section{DISCUSSION}

In the present study, under microscopic examination the $3 \mathrm{G}$ exposed livers showed swollen hepatocytes, pyknotic nuclei and vacuolations in the cytoplasm of hepatocytes, inflammatory infiltration in the liver parenchyma and around the periportal regions especially in the fourth and fifth month. Focal haemorrhages were also found within the liver parenchyma. Hepatic sinusoids were congested and dilated.

Al Galib B et al [10] reported that electromagnetic radiation emitted from mobile phone 850-1900 MHz induced inflammatory cellular infiltration around the central vein, swollen hepatocytes and vacuolated cytoplasm. Similar findings were observed by Hanafi et al [13] following exposure to $900 \mathrm{MHz}$ mobile phone radiations on mice. In a study reported by Shahram $G$ et al [16], the EMF induced coagulation necrosis, nuclear and cytoplasmic vacuolization in the rat liver; fatty liver in some rat, fibrosis of vessels along with necrosis and apoptosis of endothelium cells, dilatation of sinusoids and appearance of many phagocytic Kupffer cell. Additionally occurrence of mononuclear inflammatory cells, increased number of fibroblasts and replacement of necrotic liver cells by connective tissue were reported by Shahram G et al [16]. Laila $\mathrm{K}$ et al [11] observed the central vein and liver sinusoids congestion in rats. Cytoplasmic vacuolization and fatty accumulation were reported by Anjana V.Y et al [21] and Kamal G.I et al [22]. Robert E. Anderson [23] stated that pathogenesis of radiation induced hepatic damage could be due to injury of endothelial cells of small hepatic veins resulting venoocclusive disease.

Our study findings are consistent with the report of Al Galib et al [10], Hanafi et al [13], Shahram G et al [16], Laila K et al [11], Anjana V.Y et al [21], Kamal G.I et al [22] and Robert E Anderson [23]. However, in our study since the mice were exposed to $1900-2200 \mathrm{MHz}$ radiations for longer duration, the liver showed marked structural changes in the form of mixed type of inflammatory infiltration, focal haemorrhages, along with the appearance of cytoplasmic vacuolations and pyknotic nuclear changes.

In our study, the mean SGOT \& SGPT was found to be increased in $3 \mathrm{G}$ exposed mice in comparison with control group, however, the increase is statistically insignificant ( $p$ value $>0.05$ ). Ma HR et al [24] in their study on exposing rats to 900 $\mathrm{MHz}$ radiations, reported no significant difference in SGOT and SGPT level between experimental and control groups. Mohammed Fathy Assasa [25] observed a significant increase in SGOT and SGPT following exposure of rat to EMF from cellular telephones base stations with frequency of $900 \mathrm{MHz}$. Exposure of rats to radiofrequency radiation for 2 weeks at $40 \mathrm{KHz}$ increased approximately 3.8-fold and 2-fold higher SGPT and SGOT respectively [26]. Our study observations are consistent with Ma HR et al [24] study. Thus our study showed that, RFR emitted from $3 G$ cell phone resulted in 
histological or micro structural changes in the liver tissue, without much affecting the SGOT and SGPT levels.

Reactive oxygen species (ROS) or free radicals are the by-products of normal cellular metabolism. Free radicals are also produced by polymorphonuclear leucocytes, T-lymphocytes and macrophages to kill exogenous biological or physical materials or abnormal cells in the tissue. Free radicals production is triggered by a number of endogenous and exogenous factors and RFR emitted from a cell phone is considered one among them.

In our study, a comparison between the control and $3 G$ exposed groups showed no significant difference in the mean SOD and GPx activity. Previous studies reported an increased SOD and GPx activity in the liver on exposure to RFR. Emre et al [27], stated that, adult rats exposed to extremely low-frequency electromagnetic field showed increased SOD and GPx level in the liver. Guler $G$ et al [28] reported an increased SOD and GPx level in liver tissue following exposure to $50 \mathrm{~Hz}$ alternating electric fields in guinea pigs. Zehra Topal et al [29] also reported that, prenatal rats exposed to long duration of $900 \mathrm{MHz}$ electromagnetic fields induced an increase in SOD and GPx activity in the liver. Few contradictory findings reported decreased SOD \& GPX activity in liver, brain and kidney of rats after exposure to $900 \mathrm{MHz}$ for 30 minutes per day for 10 days [30-32]. Thus, the literature reviews have shown persistence of contradiction in the aspects of SOD and GPx levels due to RFR exposure.

Thus our study showed that, RFR emitted from $3 G$ cell phone resulted in micro structural changes in the liver tissue, without much affecting the biochemical parameters such as SGOT, SGPT, SOD and GPx levels.

\section{CONCLUSION}

Chronic exposure of mice to $1900-2200 \mathrm{MHz}$ radiations emitted from a $3 G$ mobile phone induced structural changes in the liver such as congested and dilated hepatic sinusoids, swollen hepatocytes with pyknotic nuclei, vacuolations in the cytoplasm, inflammatory infiltration and focal haemorrhages in the liver parenchyma.
Thus RFR emitted from $3 G$ cell phone results in histological or micro structural changes in the mice liver, without much affecting the SGOT, SGPT and antioxidant enzymes like SOD \& GPX levels.

\section{ACKNOWLEDGEMENTS}

The authors sincerely thank Professors and Heads of the Department of Anatomy, Pharmacology and Pathology of Mahatma Gandhi Medical College and Research Institute, Puducherry, for their whole hearted support to carry out this research work. The authors are also grateful to authors/editors/publishers of all those articles, journals and books from where the literature for this article has been reviewed and discussed.

\section{Conflicts of Interests: None}

\section{REFERENCES}

[1]. International Commission on Non-lonizing Radiation Protection. Review of the scientific evidence on dosimetry, biological effects, epidemiological observations and health consequences concerning exposure to high frequency electromagnetic fields (100 $\mathrm{KHz}$ to $300 \mathrm{GHz}$ ). Germany. ICNIRP 16/2009.

[2]. Guidelines for Limiting Exposure to Time-varying Electric, Magnetic and Electromagnetic Fields (up to $300 \mathrm{GHz}$ ). Health Physics. 1998; 74( 4) : 494-522.

[3]. D'Andrea JA, Emmerson RY, Bailey CM, Olsen RG, Gandhi OP. Microwave radiation absorption in the rat: Frequency-dependent SAR distribution in body and tail. Bioelectromagnetics. 1985; 6(2):199-206.

[4]. Lai $H$, Singh NP. Single and double strand DNA breaks in rat brain cells after acute exposure to radiofrequency electromagnetic radiation. Int J Radiat Biol.1996; 69(4):513-21.

[5]. Osman Fikret Sonmez, Ersan Odaci, Orhan Bas and Suleyman Kaplan. Purkinje cell number decreases in the adult female rat cerebellum following exposure to $900 \mathrm{MHz}$ electromagnetic field. Brain Research. 2010; 1356: 95-101.

[6]. Ashok Agarwal, Nisarg Desai, KartikeyaMakker, Alex Varghese, Rand Mouradi, Edmund Sabanegh, Rakesh Sharma. Effects of radiofrequency electromagnetic waves (RF-EMW) from cellular phones on human ejaculated semen: an in vitro pilot study. Fertil Steril.2008;1318-1325.

[7]. Meo SA, Al-DreesAM, Husain S, Khan MM, Imran $M B$. Effects of mobile phone radiation on serum testosterone in Wistar albino rats. Saudi Med J.2010; 30(8):869-73.

[8]. Ntzouni MP, Stamatakis A, Stylianopoulou F, Margaritis LH. Short- term memory in mice is affected by mobile phone radiation. Pathophysiology.2010: 25. 
[9]. Christoph Augner, Gerhard W. Hacker. Are people living next to mobile phone base stations more strained? Relationship of health concerns, self-estimated distance to base station, and psychological parameters. Indian Journal of Occupational and Environmental Medicine.2009; 13 (3):141-145.

[10]. Al-Glaib B, Al-Dardfi M, Al-Tuhami A, Elgenaidi A and Dkhil M. A technical report on the effect of electromagnetic radiation from a mobile phone on mice organs. Libyan J Med.2007; AOP: 080107:8-9.

[11]. Laila K. Hanafy, Sawsan H, Karam, AnisaSaleh. The adverse effects of mobile phone radiation on some visceral organs. Research Journal of Medicine and Medical Sciences. 2010; 5(1):95-99.

[12]. Latifa Ishaq Khayyat. The histopathological effects of an electromagnetic field on the kidney and testis of mice. EurAsian Journal of BioSciences.2011; 5:103-109.

[13]. N Hanafi, F.Eid, A.El-Dahshan.Radiation emitted from mobile phone induces amyloidosis features in some tissues of infant mice. The Egyptian Journal of Hospital Medicine.2012; 47:132-144.

[14]. Ingole IV, Ghosh SK. Cell phone radiation and developing tissues in chick embryo: A light microscopic study of kidneys. J. Anat. Soc. India.2006; 55(2):19-23.

[15]. Ingole IV, Ghosh SK.Exposure of radiofrequency radiation emitted by cell phone and mortality in chick embryo (gallus domesticus). Biomed Res. 2006; 17(3):205-210.

[16]. Shahram Gharachorlou, Amirali Ebrahimneghazd, Hamid Mirzaie. Histopathological study on electromagnetic low frequency $(50 \mathrm{~Hz})$ effects on induction of apoptosis in liver of rat. Journal of Current Research in Science. 2013; 1( 6) : 620-624.

[17]. Belyaev IY, Koch CB, Terenius O, Roxstrom Lindquist K, Malmgren LO, H Sommer W, Salford LG, Persson BR. Exposure of rat brain to $915 \mathrm{MHz}$ GSM microwaves induces changes in gene expression but not double stranded DNA breaks or effects on chromatin conformation. Bioelectromagnetics. 2006; 27(4):295-306.

[18]. Ke Yao, YiBoYu, KaiJun Wang, Juan Ye, DeQiang Lu, Huai Jiang. Absence of effect of power-frequency magnetic fields exposure on mouse embryonic lens development.Bioelectromagnetics. 2007; 28 (8):628635.

[19].Curcio G, Valentini E, Moronif,Ferrara M, De GennaroL,Bertini M. Psychomotor performance is not influenced by brief repeated exposures to mobile phones. Bioelectromagnetics. 2008; 29 (3):237241.

[20]. Hae - June Lee, Jeong - Ki Pack, Tae - Hong Kim, Nam Kim, Soo - Yong Choi, Jae - Seon Lee, Sung - Ho Kim, Yun - Sil Lee. The lack of histological changes of CDMA cellular phone - based radio frequency on rat testis. Bioelectromagnetics. 2010; 31 (7):528534.

[21]. Anjana V.Y, David G.K, Janardan K.R.Chapter 17.Cell injury and cellular adaptations. In: Anderson's Pathology (Ivan Damjanov and James Linder $10^{\text {th }}$ edition). Elsevier Inc.2009; (1): 361-366.

[22]. Kamal G.I, Rodney S.M. Chapter 57. Liver In: Anderson's Pathology (Ivan Damjanov and James Linder $10^{\text {th }}$ edition). Elsevier Inc.2009; (2):1799 1822.

[23].Robert E.Anderson, Morgan Berthrong, Louis F.Fajardo. Radiation injury. Anderson's Pathology. $10^{\text {th }}$ edition. Volume.1.Missouri. Von Hoffman Press.1996:484-512.

[24]. Ma HR, Li YY, Luo YP, Ma XL, Gong ZQ. Effect of Guilingji Capsule on the fertility, liver functions, and serum LDH of male SD rats exposed by $900 \mathrm{MHz}$ cell phone. Zhongguo Zhong Xi Yi Jie He Za Zhi. 2014 ; 34(4):4759.

[25]. Mohammed Fathy Assasa. Effect of cellular phone field on body weight, liver enzymes blood indices and role of some antioxidant in albino rats. AAMJ, 2010; 8(3).

[26]. Sakai H, Horiguchi N, Endoh D, Nakayama K, Hayashi $\mathrm{M}$. Radiofrequency radiation at $40 \mathrm{kHz}$ induces hepatic injury in Long-Evans Cinnamon (LEC) rats, an animal model for human Wilson disease. J Vet Med Sci. 2011; 73(3):299-304.

[27]. Emre M, Cetiner S, Zenier S, Unlukurt I, Kahraman I, Topcu Z. Oxidative stress and apoptosis in relation to exposure to magnetic field. Cell Biochem Biophys.2011; 59:71-77.

[28]. Guler G, Seyhan N, Aricioglu A. Effects of static and $50 \mathrm{~Hz}$ alternating electric fields on superoxide dismutase activity and TBAR's levels in guinea pigs. Gen Physiol Biophys.2006; 25:177-193.

[29]. Zehra Topal, Hatice Hanci, Tolga Mercantepe, Huseyin Serkan Erol, Osman Nuri Keles, Haydar Kaya, Sevdegul Mungan, Ersan Odaci. The effects of prenatal long-duration exposure to $900-\mathrm{MHz}$ electromagnetic field on the 21-day-old newborn male rat liver. Turk J Med Sci. 2015; 45.

[30]. F. Oktem, F. Ozguner, H. Mollaoglu, A. Koyu, and E. $\mathrm{Uz}$, “Oxidative damage in the kidney induced by 900 $\mathrm{MHz}$-emitted mobile phone: protection by melatonin," Archives of Medical Research.2005; 36, (4): 350-355.

[31]. Merhan M.R. Effect of exposure and withdrawal of $900 \mathrm{MHz}$ electromagnetic waves on brain, kidney and liver oxidative stress and some biochemical parameters in male rats. Electromagn Biol and Med. 2014. DOI:10.3109/15368378.2014.906446.

[32]. Martinez S.J, Torres -Duran P.V, Juarez Oropeza M.A. Effects of acute electromagnetic field exposure and movement restraint on antioxidant system in liver, heart, kidney and plasma of Wister rats. A preliminary report. Int. J.Radiat. Biol.2010; 86:1088-1094.

How to cite this article: N Mugunthan, J Anbalagan, $\mathrm{S}$ Meenachi, S Rajanarayanan. STRUCTURAL CHANGES OBSERVED IN MICE LIVER DUE TO CHRONIC EXPOSURE OF 1900-2200 MHZ RADIA-TIONS EMITTED FROM MOBILE PHONE. Int J Anat Res 2019;7(1.3):6254-6260. DOI: 10.16965/ijar.2018.438 